\title{
Función renal, estado de volemia y furosemida en diálisis peritoneal
}

\author{
Francisco Cirera Segura*, Jesús Lucas Martín Espejo*, Antonia Concepción Gómez Castilla**, María Án- \\ geles 0 jeda Guerrero**
}

Enfermeros/as. * Unidad de Gestión Clínica Uro-Nefrológica. H.U. Virgen del Rocío, * * Unidad de Gestión Clínica Nefrológica. H.U. Virgen Macarena. Sevilla

\section{Resumen}

El objetivo principal fue comparar la diuresis y la función renal de los pacientes incidentes en diálisis peritoneal y su evolución en función al uso de furosemida.

El objetivo secundario fue valorar si la furosemida puede mejorar el estado de volumen.

\section{Material y métodos:}

Se realizó un estudio retrospectivo, comparativo, longitudinal y multicéntrico. La muestra fueron $\mathbf{4 0}$ pacientes.

En una unidad se utilizaron diuréticos (grupo furosemida) y en otra no (grupo control). Se recogieron variables del estudio al inicio, 6 y 12 meses.

El análisis se realizó con el SPSS 19.

\section{Resultados:}

El $57,5 \%$ de los pacientes eran hombres. La edad media fue $56,45 \pm 16,2$ años. La etiología más prevalente fue la nefritis intersticial. Ningún paciente presentó efectos secundarios.

La diuresis evolucionó igual en ambos grupos, siendo superiores en el grupo furosemida sin significación.

\section{Correspondencia: \\ Francisco Cirera Segura \\ C/ Ángel Ripoll Pastor, 4-6 $\mathrm{A}$ \\ 41006 Sevilla \\ E-mail: paco.cirera@gmail.com}

No hallamos diferencias significativas a lo largo del estudio para la diuresis, filtrado glomerular, peso, índice masa corporal y ultrafiltración media. EI $40 \%$ de los pacientes del grupo control presentaron edemas a los 6 meses frente al 17,6\% del grupo furosemida. El grupo control utilizó mayor número de intercambios hipertónicos durante el primer año.

El grupo furosemida mostró presiones arteriales sistólicas y diastólicas superiores y utilizó más hipotensores aunque sin significación.

\section{Conclusiones:}

La administración de dosis bajas de diuréticos no ha mostrado diferencias significativas para la diuresis y la función renal durante el primer año en diálisis peritoneal.

Quizás por las bajas dosis, el uso de diuréticos no mejoró los parámetros relacionados con el estado de volumen.

PALABRAS CLAVE:

- DIÁLISIS PERITONEAL

- DIURÉTICOS

- FUNCIÓN RENAL RESIDUAL

Renal function, volaemia status and furosemide in peritoneal dialysis

\section{Abstract}

The main object was to compare diuresis and renal function in peritoneal dialysis incident patients and their evolution according to the use of furosemide. 
The secondary object was to evaluate whether furosemide can improve the volume status.

\section{Material and methods:}

A multicentre, longitudinal, comparative, retrospective study was carried out. The sample was 40 patients.

In one unit diuretics (furosemide group) were used and in another they were not (control group). Study variables were noted at the start, and after 6 and 12 months.

The analysis was carried out using SPSS 19.

\section{Results:}

$57.5 \%$ of the patients were men. The average age was $56.45 \pm 16.2$ yeas. The most prevalent aetiology was interstitial nephritis. No patients presented side effects.

The evolution of diuresis was the same in both groups, and was higher in the furosemide group without significance.

We did not find significant differences throughout the study for diuresis, glomerular filtration, weight, body mass index and mean ultrafiltration. $40 \%$ of the patients in the control group presented oedemas after 6 months compared to $17.6 \%$ in the furosemide group. The control group used a larger number of hypertonic exchanges during the first year.

The furosemide group showed higher systolic and diastolic blood pressure and used more hypotensors, although without significance.

\section{Conclusions:}

The administration of low doses of diuretics has not shown significant differences for diuresis and renal functions during the first year on peritoneal dialysis.

Perhaps on account of the low doses, the use of diuretics did not improve the parameters related to the volume status.

\section{KEY WORDS:}

- PERITONEAL DIALYSIS

- DIURETICS

- RESIDUAL RENAL FUNCTION

\section{Introducción}

Dentro de los cuidados de enfermería que se desarrollan en el programa de diálisis peritoneal (DP), la preservación de la función renal residual (FRR) debe ocupar un lugar preferente.

Muchos estudios longitudinales han demostrado que existe una disminución progresiva de la FRR con el tiempo en diálisis ${ }^{1}$. Esta reducción es mayor en hemodiálisis que en DP ${ }^{2,3,4}$, y aunque es de vital importancia en ambos casos y en DP contribuye a un elevado porcentaje del aclaramiento total de solutos ${ }^{5}$, su pérdida puede conllevar el abandono de la técnica por infradiálisis, especialmente en paciente con una gran superficie corporal ${ }^{6}$.

Es por tanto indispensable valorar cualquier intervención que pudiera retrasar la perdida de la FRR.

Una de estas medidas puede ser la administración de diuréticos, que es una práctica frecuente en los pacientes que inician tratamiento renal sustitutivo con $\mathrm{DP}^{7}$. Los diuréticos además, pueden contribuir a liberar la ingesta hídrica de los pacientes manteniendo un estado de volumen adecuado $^{8}$ y pueden contribuir a la conservación de la membrana peritoneal, al necesitar un menor número de intercambios hipertónicos ${ }^{9}$. Sin embargo, su uso está sujeto a controversia y no existe mucha evidencia científica sobre sus posibles beneficios ${ }^{5,6,8}$.

Por estos motivos nos planteamos como objetivo principal de nuestro estudio, comparar la diuresis y la FRR de los pacientes incidentes en DP y su evolución durante el primer año en función de la administración o no de diuréticos.

El objetivo secundario fue valorar si el uso de diuréticos mejora los parámetros relacionados con el estado de volumen de estos pacientes.

\section{Material y métodos}

Se realizó un estudio retrospectivo comparativo longitudinal en dos unidades de DP. En una de ellas se utilizaban los diuréticos (grupo furosemida) de forma protocolizada y en la otra no (grupo control).

Los criterios de inclusión fueron los siguientes: todos los pacientes incidentes en ambas técnicas, tanto DP conti- 
nua ambulatoria (DPCA) como DP automática (DPA), con un mínimo de 3 meses en la técnica, cuya diuresis fuera mayor de $500 \mathrm{cc} / 24 \mathrm{~h}$ y que utilizaran soluciones de diálisis con bicarbonato. Los criterios de exclusión fueron: pacientes cuya expectativa de vida se considerara menor de 1 año y aquellos que procedieran de otras técnicas (hemodiálisis o trasplante renal).

Se incluyeron pacientes en el estudio desde Enero de 2006 a 31 de Diciembre de 2012. La muestra total estuvo compuesta por 40 pacientes: 19 pacientes en la unidad donde recibían tratamiento con diuréticos de forma protocolizada y 21 pacientes en la unidad donde no recibían diurético.

Se recogieron las siguientes variables de la historia digital de los pacientes; al inicio del programa, así como a los 6 meses y 12 meses de seguimiento:

1. Variables demográficas: edad, género, patología de base de la enfermedad renal crónica, diabetes mellitus e índice de masa corporal (IMC).

2. Variables relacionadas con los diuréticos (Furosemida): dosis y efectos secundarios.

3. Variables relacionadas con el estado de volumen: diuresis de 24 horas, peso, edemas, tensión arterial sistólica y diastólica, número de hipotensores exceptuando los diuréticos, ultrafiltración media.

4. Variables relacionadas con datos analíticos: sodio en sangre y orina, potasio en sangre y orina, albúmina en sangre, filtrado glomerular (eGFR por Padequest $\AA$ ).

5. Variables relacionadas con la técnica: $\mathrm{Kt} / \mathrm{V}$ peritoneal, renal y total, test de equilibrio peritoneal (TEP), uso de líquidos hipertónicos (considerando hipertónico todo concentración de glucosa mayor de $1,36 \%$, incluyendo la icodextrina) y número de infecciones peritoneales.

Los pacientes tratados con furosemida tomaban una dosis media de 82,10 mg/24 horas por vía oral, (40-240).

El análisis de datos se realizó con el paquete estadístico IBM SPSS Statistics 19.0, estableciendo el nivel de significación en $p<0,05$. Para el estudio de las variables cualitativas usamos frecuencias absolutas y relativas, y para las cuantitativas utilizamos media y la desviación estándar o la mediana y el rango intercuartílico respectivamente. Para a comparación de las características según los grupos se usaron la chi-cuadrado (para variables cualitativas) y la t-student o la U de Mann-Whitney (para las cuantitativas).

\section{Resultados}

La muestra la compusieron un total de 40 pacientes, el $57,5 \%$ eran hombres $(n=23)$. La edad media fue 56,45 $\pm 16,20$ años. El $50 \%(n=20)$ estaban en DPCA. La etiología de la enfermedad renal crónica se muestra en la Tabla 1, se analizó también si existían mayor prevalencia de nefritis intersticiales en alguno de los grupos, ya que conllevan un menor deterioro de la función renal, no existiendo diferencias $(p=0,712)$.

\begin{tabular}{|c|c|c|c|}
\hline & Control & Furosemida & Total \\
\hline $\begin{array}{c}\text { Pielonefritis/ } \\
\text { Nefritis Intersticial }\end{array}$ & $19 \%(n=4)$ & $26,3 \%(n=5)$ & $22,5 \%(n=9)$ \\
\hline $\begin{array}{l}\text { Enfermedades } \\
\text { Vasculares }\end{array}$ & $23,8 \% \quad(n=5)$ & $15,8 \%(n=3)$ & $20 \%(n=8)$ \\
\hline No Filiada & $14,3 \%(n=3)$ & $21,1 \%(n=4)$ & $17,5 \%(n=7)$ \\
\hline Glomerulonefritis & $14,3 \%(n=3)$ & $10,5 \%(n=2)$ & $12,5 \%(n=5)$ \\
\hline Nefropatía Diabética & $9,5 \%(n=2)$ & $15,8 \%(n=3)$ & $12,5 \%(n=5)$ \\
\hline $\begin{array}{l}\text { Enfermedad } \\
\text { Poliquística }\end{array}$ & $9,5 \%(n=2)$ & $10,5 \%(n=2)$ & $10 \%(n=4)$ \\
\hline $\begin{array}{l}\text { Enfermedades } \\
\text { Sistémicas }\end{array}$ & $9,5 \%(n=2)$ & $0,00 \%$ & $5 \%(n=2)$ \\
\hline
\end{tabular}

Tabla 1. Etiología de la Enfermedad Renal Crónica*.

Códigos EDTA 1994-1995 y su agrupación, extraídos del Informe 2006. Módulo Básico. Subsistema de Insuficiencia Renal Crónica. Servicio Andaluz de Salud. Junta de Andalucía.

Los datos demográficos y analíticos de ambos grupos, así como las principales características relacionadas con el estado de volumen y la técnica de diálisis se compararon al inicio del estudio (Tabla 2), no encontrando diferencias significativas en ningún caso, excepto en el $\mathrm{Kt} / \mathrm{V}$ renal $(p=0,004)$ y el potasio en orina $(p=0,007)$, pudiendo considerarse iguales al inicio del estudio. Los grupos también eran iguales en la distribución de los

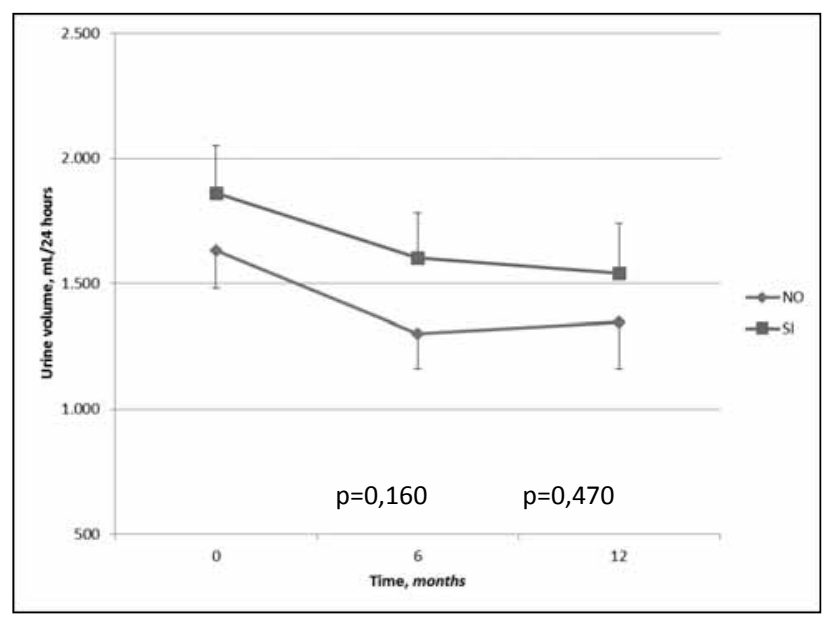

Figura 1. Evolución de la diuresis en función del uso de furosemida. Datos representados Media + DE. p: T de Student. Diuresis de 24 horas en mililitros. Tiempo de evolución en meses. 


\begin{tabular}{|c|c|c|c|c|}
\hline & & Control & Furosemida & $\mathbf{p}$ \\
\hline \multicolumn{2}{|c|}{ Pacientes } & 21 & 19 & \\
\hline \multicolumn{2}{|c|}{${ }^{1}$ Hombres : Mujeres } & $14: 7$ & $9: 10$ & 0,22 \\
\hline \multicolumn{2}{|c|}{${ }^{2}$ Edad (años) } & $54,67 \pm 15,73$ & $58,42 \pm 16,91$ & 0,47 \\
\hline \multicolumn{2}{|c|}{${ }^{1}$ Diabetes Mellitus (Si) } & 6 & 7 & 0,58 \\
\hline \multicolumn{2}{|c|}{${ }^{1}$ Modalidad (DPA) } & 11 & 9 & 0,76 \\
\hline \multicolumn{2}{|c|}{${ }^{3}$ Numero Infecciones Peritoneales } & $0[0-0,5]$ & $0[0-1]$ & 0,86 \\
\hline \multirow{2}{*}{ Diuresis } & ${ }^{2}$ Orina 24 Horas $(\mathrm{mL})$ & $1632,5 \pm 677,7(n=20)$ & $1862,1 \pm 749,26$ & 0,32 \\
\hline & ${ }^{2}$ eGFR & $6,8 \pm 2,6(n=20)$ & $7,8 \pm 3,5$ & 0,34 \\
\hline \multirow{3}{*}{ Dosis de Diálisis } & ${ }^{3} \mathrm{Kt} / \mathrm{V}$ Total & $2,58[2,14-3,06]$ & $2,72[2,41-3,11]$ & 0,33 \\
\hline & ${ }^{3}$ Kt/V Renal & $1,08[0,72-1,5]$ & $1,7[1,07-2,02]$ & 0,05 \\
\hline & ${ }^{2} \mathrm{Kt} / \mathrm{V}$ Peritoneal & $1,47 \pm 0,24(n=17)$ & $1,20 \pm 0,27$ & 0,004 \\
\hline \multirow{6}{*}{$\begin{array}{l}\text { Estado de } \\
\text { Volumen }\end{array}$} & ${ }^{1}$ Edemas (SI) & 5 & 1 & 0,19 \\
\hline & ${ }^{3} \mathrm{~N}^{0}$ de hipotensores & $2[1-3]$ & $1[0-2]$ & 0,06 \\
\hline & ${ }^{2}$ Peso $(\mathrm{Kg})$ & $71,09 \pm 12,92$ & $74,97 \pm 18,03$ & 0,43 \\
\hline & ${ }^{3}$ UF media & $585[200-862]$ & $641[334-854]$ & 0,61 \\
\hline & ${ }^{2}$ PA Sistólica & $136,86 \pm 19,42$ & $140,84 \pm 17,96$ & 0,51 \\
\hline & 2PA Diastólica & $81,95 \pm 11,67$ & $88,47 \pm 12,26$ & 0,09 \\
\hline \multirow{5}{*}{ Analítica } & 2Álbumina & $3,89 \pm 0,43(n=20)$ & $3,84 \pm 0,39$ & 0,71 \\
\hline & ${ }^{3}$ Sodio & $140[137,5-143,5]$ & 142 [139-144] & 0,36 \\
\hline & ${ }^{2}$ Potasio & $5,03 \pm 0,91$ & $4,95 \pm 0,79$ & 0,78 \\
\hline & ${ }^{3}$ Potasio Orina & $20[13,5-23,5]$ & $12[8,75-19,25]$ & 0,007 \\
\hline & ${ }^{2}$ Sodio Orina & $70,76 \pm 19,99(n=17)$ & $74,21 \pm 29,39(n=14)$ & 0,70 \\
\hline
\end{tabular}

Tabla 2. Comparación de las características basales según la utilización de furosemida.

Datos representados Mediana [p25-p75] o Media + DE. 1Test Exacto de Fisher. 2T de Student. 3U de Mann Whitney. eGFR: Filtrado Glomerular. UF: Ultrafiltración. PA: Presión Arterial.

pacientes respecto al test de equilibrio peritoneal. Ningún paciente presentó efectos secundarios al uso de la furosemida.

La diuresis de 24 horas evolucionó de forma similar en ambos grupos durante el primer año en DP (figura 1), con un descenso inicial hasta los seis meses que se modera en el grupo de la furosemida y mejora en el grupo de control, aunque siempre son superiores las diuresis del grupo que utiliza la furosemida sin llegar a ser signi- ficativo ni a los seis meses $(1302,5 \pm 141,6$ vs $1602,9 \pm$ $154,5 ; p=0,160)$ ni a los doce meses $(1345 \pm 183,6$ vs $1539,3 \pm 191,7 ; p=0,470$ ).

Estudiamos la evolución de los parámetros relacionados con la función renal residual y el estado de volumen. Los resultados se muestran en la tabla 3, no hallando significación a los seis ni a los doce meses en la diuresis, el filtrado glomerular, el peso, el IMC y la ultrafiltración media.

\begin{tabular}{|c|c|c|c|c|c|c|}
\hline & \multicolumn{3}{|c|}{6 Meses } & \multicolumn{3}{|c|}{12 Meses } \\
\hline & Control & Furosemida & p & Control & Furosemida & p \\
\hline Diuresis $(\mathrm{mL})$ & $\begin{array}{c}-300 \\
{[-1000 ; 100]}\end{array}$ & $\begin{array}{c}\mathbf{0} \\
{[-1100 ; 75]}\end{array}$ & $836^{2}$ & $\begin{array}{c}-458,93 \\
\pm 673,08\end{array}$ & $\begin{array}{c}-480,71 \\
\pm 778,20\end{array}$ & , $937^{1}$ \\
\hline eGFR & $\begin{array}{c}-1,17 \\
+2,75\end{array}$ & $\begin{array}{c}-0,77 \\
+2,99\end{array}$ & 6871 & $\begin{array}{c}-2,83 \\
{[-4,28 ;-1,69]}\end{array}$ & $\begin{array}{c}-1,55 \\
{[-4,28 ; 0,38]}\end{array}$ &, $332^{2}$ \\
\hline Peso $(\mathrm{Kg})$ & $\begin{array}{r}1,5 \\
+3,18\end{array}$ & $\begin{array}{c}1,58 \\
\pm 2,57\end{array}$ &, $942^{1}$ & $\begin{array}{c}1,5 \\
{[0,15 ; 2,85]}\end{array}$ & $\begin{array}{c}2,25 \\
{[0,17 ; 5,1]}\end{array}$ &, $323^{2}$ \\
\hline IMC & $\begin{array}{c}0,54 \\
\pm 1,17\end{array}$ & $\begin{array}{c}0,60 \\
\pm 0,94\end{array}$ & $861^{1}$ & $\begin{array}{c}0,47 \\
\pm 0,75\end{array}$ & $\begin{array}{c}1,12 \\
\pm 1,38\end{array}$ & $137^{1}$ \\
\hline UF media $(m L)$ & $\begin{array}{c}109,1 \\
\pm 466,9\end{array}$ & $\begin{array}{c}69,52 \\
+439,1\end{array}$ &, $796^{1}$ & $\begin{array}{c}318,21 \\
+367,19\end{array}$ & $\begin{array}{c}266,5 \\
+523,51\end{array}$ &, $765^{1}$ \\
\hline
\end{tabular}

Tabla 3. Evolución de los parámetros relacionados con el estado de volumen.

Datos representados Mediana [p25;p75] o Media + DE. IT de Student. 2U de Mann Whitney. eGFR: Filtrado Glomerular. UF: Ultrafiltración. IMC: Índice de masa corporal. 
El $40 \%(n=8)$ de los pacientes del grupo control presentaron edemas a los seis meses frente a $17,6 \%(n=3)$ del grupo furosemida $(p=0,138)$ a los doce meses fueron $13,3 \%$ $(n=2)$ vs $15,4 \%(n=2)$, sin significación $(p=0,999)$.

Se analizó el uso de líquidos hipertónicos en ambos grupos, encontrando que el grupo que no utilizaba furosemida utilizó mayor número de intercambios con líquidos hipertónicos tanto a los seis meses donde el $63,2 \%(n=12)$ de los pacientes utilizaba líquidos hipertónicos habitualmente frente al $10,5 \%(n=2)$ del grupo de la furosemida, como a los doce meses $(68,8 \%(n=11)$ vs $5,3 \%(n=1))$
Valoramos también el efecto que tuvo el uso de diuréticos sobre el control de la presión arterial en los pacientes en programa de DP, como se muestra en la tabla 4. El grupo que utilizó furosemida mostró presiones arteriales sistólicas más elevadas a los 6 meses y a los doce meses, lo mismo ocurrió con las diastólicas, aunque en ambos casos no se halló significación. El grupo de la furosemida también utilizaba más hipotensores a los 6 meses y a los doce meses, aunque sin significación. Tampoco se encontraron diferencias para el sodio en orina ni para la albuminemia a lo largo del estudio.

\begin{tabular}{|c|c|c|c|c|c|c|}
\hline & \multicolumn{3}{|c|}{6 Meses } & \multicolumn{3}{|c|}{12 Meses } \\
\hline & Control & Furosemida & p & Control & Furosemida & p \\
\hline $\begin{array}{l}\text { Número de } \\
\text { Hipotensores }\end{array}$ & $\begin{array}{c}1 \\
{[0-2]}\end{array}$ & $\begin{array}{c}2 \\
{[1-2,5]}\end{array}$ & $108^{2}$ & $\begin{array}{c}1 \\
{[0-2]}\end{array}$ & $\begin{array}{c}1 \\
{[1-2]}\end{array}$ & $217^{2}$ \\
\hline P.A. Sistólica & $\begin{array}{r}127,7 \\
\pm 24,4\end{array}$ & $\begin{array}{l}136,4 \\
\pm 14,9\end{array}$ & $208^{1}$ & $\begin{array}{c}125 \\
{[115 ; 150]}\end{array}$ & $\begin{array}{c}133,5 \\
{[121,7 ; 146,2]}\end{array}$ &, $347^{2}$ \\
\hline P.A. Diastólica & $\begin{array}{r}75,2 \\
+14,2\end{array}$ & $\begin{array}{r}79,8 \\
+9,4\end{array}$ & $252^{1}$ & $\begin{array}{c}\mathbf{8 2 , 4} \\
+14,5\end{array}$ & $\begin{array}{r}85,8 \\
+19\end{array}$ &, $594^{1}$ \\
\hline P.A. Media & $\begin{array}{l}92,7 \\
\pm 16\end{array}$ & $\begin{array}{r}98,6 \\
+8,5\end{array}$ & $160^{1}$ & $\begin{array}{c}98,1 \\
+13,9\end{array}$ & $\begin{array}{l}103,6 \\
\pm 20\end{array}$ &, $400^{1}$ \\
\hline Sodio en Orina & $\begin{array}{c}68 \\
+36,8\end{array}$ & $\begin{array}{c}78,4 \\
+27,1\end{array}$ &, $386^{1}$ & $\begin{array}{c}83,2 \\
\pm 27,1\end{array}$ & $\begin{array}{c}77,8 \\
+26,2\end{array}$ & $633^{1}$ \\
\hline Albúmina & $\begin{array}{c}3,7 \\
{[3,6 ; 3,95]}\end{array}$ & $\begin{array}{c}4 \\
{[3,6 ; 4,25]}\end{array}$ &, $145^{2}$ & $\begin{array}{c}3,7 \\
\pm 0,3\end{array}$ & $\begin{array}{c}4,0 \\
+0,4\end{array}$ &, $035^{1}$ \\
\hline
\end{tabular}

Tabla 4. Control de la presión arterial según el uso de furosemida.

Datos representados Mediana [p25;p75] o Media + DE. 1T de Student. 2U de Mann Whitney. PA: presión arterial.

Se analizaron también el sodio plasmático $(140,6 \pm$ 3,13 vs $142,47 \pm 4,29 ; p=0,135$ y $140,6 \pm 2,95$ vs $141,9 \pm 5,24 ; p=0,403)$ y el potasio $(5,07 \pm 0,78$ vs $5,36 \pm 0,81 ; p=0,266$ y $4,93 \pm 0,84$ vs $5,16 \pm 0,61 ;$ $p=0,423$ ) siendo ambos más elevados en el grupo de la furosemida aunque sin significación en ningún caso.
Por último, estudiamos si el uso de la furosemida tuvo algún tipo de repercusión sobre la dosis de diálisis administrada como se muestra en la tabla 5, no existiendo diferencias entre los grupos.

\begin{tabular}{|c|c|c|c|c|c|c|}
\hline & \multicolumn{3}{|c|}{6 Meses } & \multicolumn{3}{|c|}{12 Meses } \\
\hline & Control & Furosemida & p & Control & Furosemida & p \\
\hline Kt/V Total & $2,59 \pm 0,9$ & $2,67 \pm 0,59$ & ,754 & $\begin{array}{c}2,5 \\
{[1,94 ; 2,88]}\end{array}$ & $\begin{array}{c}2,42 \\
{[2,09 ; 3,04]}\end{array}$ &, $800^{2}$ \\
\hline Kt/V Renal & $1,24 \pm 0,8$ & $1,5 \pm 0,7$ & $276^{2}$ & $1,1 \pm 0,6$ & $1,3 \pm 0,7$ &, $448^{2}$ \\
\hline Kt/V Peritoneal & $\begin{array}{c}1,32 \\
{[1,19 ; 1,52]}\end{array}$ & $\begin{array}{c}1,13 \\
{[1,01 ; 1,23]}\end{array}$ &, $017^{2}$ & $\begin{array}{c}1,42 \\
{[1,26 ; 1,54]}\end{array}$ & $\begin{array}{c}1,14 \\
{[0,97 ; 1,43]}\end{array}$ &, $077^{2}$ \\
\hline
\end{tabular}

Tabla 5. Adecuación de la diálisis y diuréticos.

Datos representados Mediana [p25;p75] o Media + DE. 1T de Student. 2U de Mann Whitney.

\section{Discusión}

En diálisis peritoneal, la preservación de la función renal residual es tan importante como la viabilidad de la membrana peritoneal a largo plazo ${ }^{10}$, ya que ha demostrado tener repercusión directa sobre la supervivencia y ha demostrado ser un factor independiente en estudios longitudinales ${ }^{11}$.
Estudios como el CANUSA han demostrado que un incremento de $0,5 \mathrm{~mL} / \mathrm{min}$ de filtrado glomerular se asocia a un descenso del $9 \%$ de riesgo de muerte ${ }^{11}$ y que un incremento de $1 \mathrm{ML} / \mathrm{Min}$ es comparable a un intercambio diario de 2 litros, a un Kt/V semanal de $0,25-0,3$ o an aclaramiento de creatinina de 10 litros ${ }^{12}$. 
Por estos motivos, cualquier medida dirigida a mantener la función renal residual debe ser evaluada exhaustivamente.

Diversos trabajos han planteado el uso de la furosemida en los pacientes en diálisis peritoneal, para prolongar la función renal residual, obteniendo diversos resultados. Algunos de ellos afirman que el uso de diuréticos a altas dosis es útil para prevenir la ganancia de peso debida a la retención de agua evitando el uso de líquidos hipertónicos ${ }^{2}$. Los mismos resultados comunicó Scarpioni et $\mathrm{al}^{8}$, con dosis de furosemida de $500 \mathrm{mg} /$ diarios, aunque al año los pacientes se hicieron resistentes.

Ambos estudios con dosis de furosemida tan elevadas pueden considerarse cuasi experimentales, ya que esas dosis en la práctica clínica habitual tienen el riesgo de ototoxicidad y de crear resistencias a la furosemida perdiendo una herramienta útil para el control de la volemia en caso necesidad, así como de influir en la adherencia al tratamiento ya que implican tomar un numero de comprimidos diarios muy elevado.

Por tanto, estos resultados hallados en la bibliografía no parecen respaldar la furosemida como protector de la función renal, aunque sí que podría ayudar a mantener el equilibrio hídrico de los pacientes en diálisis peritoneal ${ }^{13}$, ya que pueden doblar la diuresis residual, acompañándose de un aumento de la excreción de sodio, cloro y potasio siempre que se utilice a dosis altas ( $1000 \mathrm{mg} /$ día) como corroboran diversos trabajos ${ }^{3,8}$.

En nuestro estudio, los grupos pueden considerarse iguales tras analizar las características basales. Hay que destacar como primera causa de la enfermedad renal crónica, la nefropatía intersticial a diferencia de lo que se publica en los últimos registros nacionales, lo que puede deberse a que esta patología mantiene la función renal durante un mayor tiempo y por tanto los es susceptible de mantenerse con el uso de furosemida.

Aunque sin significación estadística, el grupo furosemida inició el tratamiento sustitutivo con diálisis peritoneal con un mayor filtrado glomerular, por protocolo del servicio, lo que puede explicar la mayor diuresis de este grupo durante el primer año, así como la menor dosis de diálisis peritoneal y el menor uso de líquidos hipertónicos, aunque siempre sin significación. Por otro lado en nuestro estudio hemos analizado también la evolución de estas variables durante el primer año, no hayando diferencias tampoco.
A diferencia de lo descrito en la bibliografía, la furosemida no ha reportado beneficios en el control del estado de volumen de los pacientes. No obstante nos ha llamado la atención que en el grupo furosemida se utilizarán incluso más hipotensores, teniendo tensiones arteriales sistólicas y diastólicas más elevadas y usaban menos líquidos hipertónicos, pudiendo deberse a una decisión clínica de tener a los pacientes con una volemia superior, y de una mayor restricción del uso de líquidos hipertónicos como medidas para mantener la función renal y preservar la membrana peritoneal.

Quisiéramos destacar alguna de las limitaciones del estudio como pudiera ser que las dosis de furosemida administradas son muy inferiores a las descritas en otros trabajos, lo que podría justificar que no existieran diferencias entre los grupos. De igual forma, que cada grupo de estudio coincida estrictamente con dos unidades distintas podría influir en los resultados ya que los criterios de entrada en diálisis así como del tratamiento administrado pudieran ser distintos, en lo que respecta a la función renal y su conservación.

Podemos concluir que el uso de la furosemida, a las dosis que las usamos en nuestros pacientes, no ha tenido ninguna repercusión sobre el mantenimiento de la función renal de los pacientes incidentes en diálisis peritoneal. Tampoco hemos observado que contribuya a un mejor control del estado de volumen de los pacientes en DP.

Recibido: 20 Octubre 2013

Revisado: 2 noviembre 2013

Modificado: 10 Noviembre 2013

Aceptado: 12 Noviembre 2013

\section{Bibliografía}

1. Rottemburgo J, Issad B, Gallego JL et al. Evolution of residual renal function in patients undergoing maintenance haemodialysis or continuous ambulatory peritoneal dialysis. Proc EDTA 1982; 19:397-402.

2. Lameire N, Van Biesen W. The impact of residual renal function on the adequacy of peritoneal dialysis. Perit Dial Int. 1997; 17(suppl 2):S102S110. 
3. James $F$, Medcalf $K$, Harris PG, Walls J. Role of diuretics in the preservation of residual renal function in patients on continuous ambulatory peritoneal dialysis. Kidney Int 2001; 59:1128-1133.

4. Lang S, Bergner A, Topfer M, Schiff H. Preservation of residual renal function in dialysis patients: effects of dialysis-technique-related factors. Perit Dial Int 2001; 21(1):52-58.

5. Heimburguer 0. Residual renal function, peritoneal transport characteristics and dialysis adequacy in peritoneal dialysis. Kidney Int 1996; 50(suppl 56):S47-S55.

6. Díaz-buzo JA, Gotch FA, Folden TI et al. Peritoneal dialysis adequacy: a model to assess feasibility with various modalities. Kidney Int. 1999; 55:2493-2501.

7. Guías SEN. Guías de práctica clínica en diálisis peritoneal de la SEN. Nefrología 2006; 26 (4).

8. Scarpioni I. Ballochi S, Bergonzi $G$ et al. High-dose diuretics in continuous ambulatory peritoneal dialysis. Perit Dial Int. 1982; 177-178.
9. Kam Tao Li P, Lun Cheng Y. Therapeutic options for preservation of residual renal function in patients on peritoneal dialysis. Perit Dial Int 2007; 27 (suppl 2):S158-S163

10. Tam, P. Peritoneal dialysis and preservation of residual renal function. Perit. Dial Int 2009; (suppl 2):S108-S110.

11. Canadá-usa (CANUSA) peritoneal dialysis study group. Adequacy of dialysis and nutrition in continuous peritoneal dialysis: association with clínical outcomes. J Am Soc. Nephrol 1996; 7:198-207.

12. Li PK, Clown KM. The cost barrier to peritoneal dialysis in the developing world. An Asian perspective. Perit Dial Int 2001; 21(suppl 3):S307S313.

13. Menon MK, Naimark DM, Bargman JM, Vas SI, Oreopoulos DG. Long-term blood pressure control in a cohort of peritoneal dialysis patients and its association with residual renal function. Nephrol Dial Trasplant 2001; 16:2207-13. 